\title{
An Improvement of the Piggyback Algorithm for Parallel Model Checking
}

\author{
Ioannis Filippidis \\ Control and Dynamical Systems \\ California Institute of Technology \\ Pasadena CA 91125, USA \\ ifilippi@caltech.edu
}

\author{
Gerard J. Holzmann \\ Laboratory for Reliable Software \\ Jet Propulsion Laboratory \\ California Institute of Technology \\ Pasadena CA 91109, USA \\ gerard.j.holzmann@jpl.nasa.gov
}

\begin{abstract}
This paper extends the piggyback algorithm to enlarge the set of liveness properties it can verify. The original algorithm is based on parallel breadth-first search and piggybacking of accepting states. Its extension is motivated by an attempt to express in logic the counterexamples it can detect, relating them to bounded liveness. Piggybacked accepting states are deleted whenever a counter of transitions expires. The main improvement is obtained by renewing this counter when the same accepting states are visited in the negated property automaton. Piggybacking multiple states is considered and a memory-efficient variant that stores piggybacked states in Bloom filters. Bounded suffix detection in parallel is discussed, in which local searches attempt to connect fragmented cycles.
\end{abstract}

\section{General Terms}

Model Checking, Linear Temporal Logic

\section{Keywords}

Parallel, Piggyback, Breadth-First Search

\section{INTRODUCTION}

Over the past 30 years model checking [5] has benefited from the increase of processor clock speed, as described by Moore's law. However limits on existing technology have significantly decelerated this rate in the past ten years. To compensate, the industry has shifted its focus to pursuing improvements through a change of computation model from single to multiple processor architectures. Considering how the transition of everyday computing to multiple cores reflects in supercomputing platforms and Graphics Processing Units (GPUs) motivates exploiting parallel computing facilities by adapting existing algorithms. How effective parallelization can be in reducing an algorithm's computation time depends on the algorithm's complexity class and is considered in section 8 .

There has been a considerable amount of work on paral- lel model checking algorithms [6], with several approaches not based on depth-first search (DFS). Nested DFS was distributed over dual core machines in [11] and multiple cores in [16]. A review of several methods, including negative cycle detection, computing accepting predecessors, exploiting back-level edges of the BFS tree, an approach based on strongly connected components (SCC) and use of dependency graph data structures can be found in [6]. These approaches are distributed over clusters and need to partition the state space accordingly, whereas in [12] and here a shared memorv implementation is considered. The method proposed in [24] to translate properties from liveness to safety and augment transition systems to detect when a cycle closes by matching a recorded state has similarities with piggybacking states, although the former requires that an oracle be available.

The paper is organized as follows. The original piggyback algorithm is reviewed in section 2, its relation to bounded liveness investigated in section 3 and its incompatibility with negation shown in section 4 . The limitations of the original algorithm are outlined in section 5 to motivate the modifications introduced in section 6, which describes the extended algorithm and discusses bounded-suffix LTL properties, which are supported by the experimental results presented in section 7. In section 8 results from parallel computational complexity theory are invoked to motivate the attempts to parallelize model checking. Conclusions are summarized in section 9 .

\section{PIGGYBACK ALGORITHM}

The widely used nested depth-first search algorithm for LTL model checking [13] is not expected to be highly parallelizable [22]. In [12] a parallel breadth-first search algorithm was proposed for the verification of safety properties as well as an extension called the piggyback algorithm for a subset of liveness properties, and both were implemented in the SPIN model checker [10]. In each iteration the algorithm advances the BFS front by one step, by distributing the generation of successor states among processors while avoiding locks, as shown in Fig. 1a. Each core (middle) processes its own input queue (yellow/up) and distributes the successors uniformly randomly to its own output queues (green/bottom). Next each output queue will become part of another core's input queue, as the processing direction is toggled (arrow flipped). This input/output orthogonality coupled with uniform randomization are crucial in ensuring 


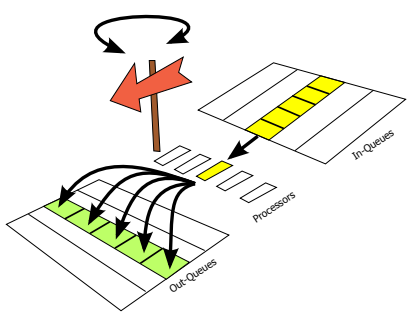

(a) Two sets of queues.

Figure 1: Original piggyback algorithm [12].

uniform effort distribution, minimizing idle time. The safety version terminates either when an accepting state of the finite automaton is reached, or when the reachable state space has been covered without reaching any accepting state. In contrast, the liveness version has to detect accepting cycles. Instead of performing a nested search, cycles are detected by carrying around (piggybacking) the last accepting state encountered along each search path, which emulates a partial nested search. In [12] the piggybacked accepting state is dropped after an a priori fixed upper bound on iterations since it was discovered, as schematically shown in Fig. 1b. Thus only a subset of liveness properties could be verified, because the nested search is restricted to a subset of states reachable from each accepting state. Here we extend this subset and improve on its description. The term boundedsuffix model checking refers to this bound on the suffix, and differs from bounded model checking [7] in that the prefix and suffix are not bounded a priori. A key observation is that the search is performed in the synchronous product $\mathcal{T} \otimes \mathcal{A}_{\neg \varphi}$ of the transition system (TS) $\mathcal{T}$ with the negated property Büchi Automaton (BA) $\mathcal{A}_{\neg \varphi}$. So the cycle bound is enforced in $\mathcal{T} \otimes \mathcal{A}_{\neg \varphi}$, not in $\mathcal{A}_{\neg \varphi}$, a distinction whose importance will be shown later.

We use the following definitions. The term property will refer interchangeably to either the desired LTL formula $\forall \varphi$ or its associated Büchi Automaton $\mathcal{A}$. A never claim refers to the negation of the desired formula, i.e., $\exists \neg \varphi$, or the equivalent BA $\mathcal{A}_{\neg \varphi}$. A product state is a state of the synchronous product $\mathcal{T} \otimes \mathcal{A}_{\neg \varphi}$. For each accepting state $q \in F_{\mathcal{A}_{\neg \varphi}}$ we define its acceptance group $\operatorname{acc}(q)$ as the subset of states $\left\{\left(s_{i}, q_{j}\right) \in \mathcal{T} \otimes \mathcal{A}_{\neg \varphi} \mid q_{j}=q\right\}$. Each accepting group defines an equivalence class, so $\cup_{q \in F_{\mathcal{A}_{\neg \varphi}}} \operatorname{acc}(q)$ form a partition of the accepting product states $F_{\mathcal{T} \otimes \mathcal{A}_{\neg \varphi}}$.

\section{RELATION TO METRIC TL}

The first question we explore is whether for a given LTL formula $\varphi$ there exists another formula $\psi$ that describes the set of TS traces accepted by the piggyback algorithm with input $\varphi$ and $\mathcal{T}$. Metric Temporal Logic (MTL) was introduced by Koymans [14] to enable quantitative reasoning about time, as opposed to only qualitative, as with LTL. Time interpretation can be discrete or dense, the latter causing partial undecidability [9]. MTL includes the bounded operators $\square_{k}, \diamond_{k}$ in addition to $\square, \mathcal{U}$. Over $\mathbb{N}$-time MTL is fully decidable. By identifying time with indices in the state sequence, the operators $\square_{k}, \diamond_{k}$ can be expressed using a bounded number of next operators $\bigcirc \bigcirc \ldots \bigcirc$. The conventional definition (p.391 [3], [19]) for an $\omega$-word over alphabet $\Sigma \triangleq 2^{P}$ at $i \in \mathbb{N}$ is $(w, i) \models \diamond_{k} \varphi$ iff $\exists j$ such that $i \leq j \leq i+k$ and $(w, j) \models \varphi$. The corresponding temporal operators are

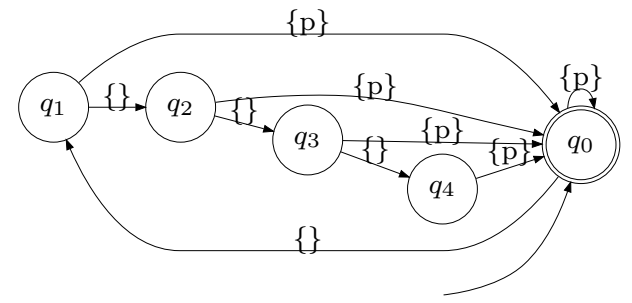

Figure 2: $\mathcal{A}_{\neg \varphi}$ corresponding to $\neg \varphi=\square \diamond_{3} p$ with next operators expanded.

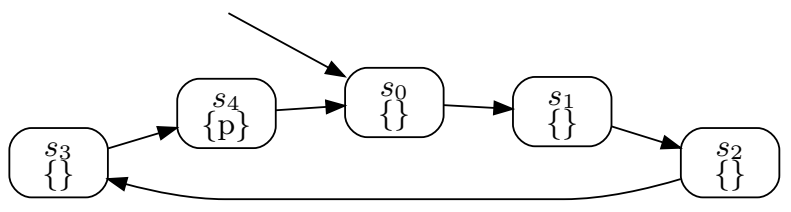

(a) Transition system $\mathcal{T}$ with labeling injective with respect to $\{p\}$.

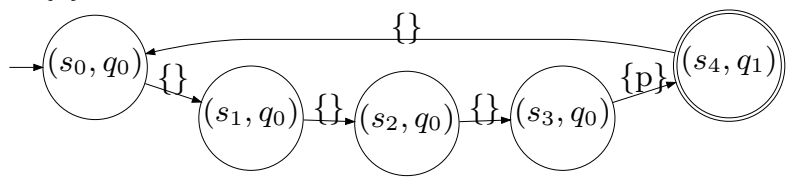

(b) Product $\mathcal{T} \otimes \mathcal{A}_{\neg \varphi}$ for $\mathcal{T}$ of Fig. 3a and $\neg \varphi=\square \diamond p$ (Fig. 5b).

Figure 3: Partially-injective labeling $L$ with respect to $\{p\}$, with product automaton shown in Fig. $5 \mathrm{~b}$.

$\diamond_{k} \varphi \triangleq \varphi \vee \bigvee_{i=1}^{k} \bigcirc_{i} \varphi$ where $\bigcirc_{i+1} \triangleq \bigcirc_{i}$ and $\bigcirc_{1} \triangleq \bigcirc$, and $\varphi \mathcal{U}_{k} \psi \triangleq(\varphi \mathcal{U} \psi) \wedge\left(\diamond_{k} \psi\right)$, similarly for $\square_{k}$. Expressing timed properties in expanded "next" form leads to larger automata, e.g., $\square \diamond_{3} p$ in Fig. 2. Timed Büchi automata offer a more compact representation, proving conceptually useful later.

\subsection{Label injectivity}

This section proves that it is in general impossible to express the set of traces accepted by the original piggyback algorithm using logic. If the labeling function $L: S \rightarrow 2^{A P}$ is not injective, then it may project multiple states of the product automaton $\mathcal{T} \otimes \mathcal{A}_{\neg \varphi}$ on the same accepting state of $\mathcal{A}_{\neg \varphi}$. In Fig. 4 all states are labeled with $\{p\}$, so the BA for $\neg \varphi=\square \diamond_{1} p$ remains at its accepting state, because it observes uninterruptedly $\{p\}$. In contrast the piggyback algorithm expects to match the same state in $\mathcal{T} \otimes \mathcal{A}_{\neg \varphi}$. But the cycle in $\mathcal{T} \otimes \mathcal{A}_{\neg \varphi}$ has length 3 , so for $k=1$ the algorithm fails to find the accepting cycle. Thus the piggyback algorithm makes a finer distinction between states in order to match an accepting state and detect a cycle. So a ver-

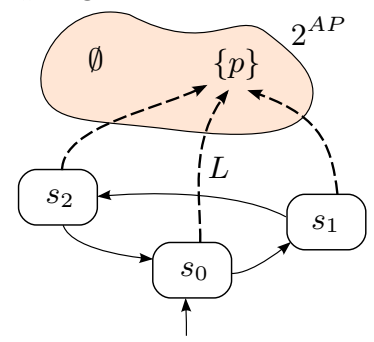

Figure 4: A labeling $L$ that is not injective wrt $\{p\}$. 


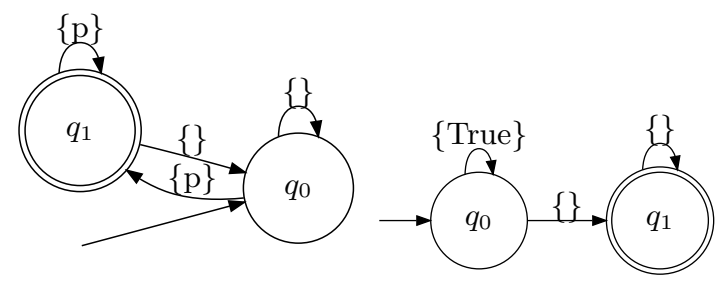

(a) $\square \diamond p$

(b) $\diamond \square \neg p$

Figure 5: Büchi automata for recurrence property and its negation.

sion of $\neg \varphi$ with eventualities bounded does not correspond to the piggyback algorithm. The difference arises from placing the bound on accepting cycles of $\mathcal{A}_{\neg \varphi}$ when converting $\varphi$ eventualities from LTL to MTL. Instead, the piggyback algorithm places the bound on accepting cycles of $\mathcal{T} \otimes \mathcal{A}_{\neg \varphi}$, which do not correspond to those in $\mathcal{A}_{\neg \varphi}$ when $L$ is not injective. For an injective $L$ the completion of an accepting cycle in $\mathcal{A}_{\neg \varphi}$ implies the recurrence of label $\{p\}$. Since a unique state of $\mathcal{T}$ is labeled with $\{p\}$, recurrence of $\{p\}$ implies that $\mathcal{T}$ closes a cycle. This ensures that both $\mathcal{A}_{\neg \varphi}$ and $\mathcal{T}$ close an accepting cycle simultaneously, as in Fig. 3. However, this is not the case when lack of (partial) injectivity wrt $\{p\}$ leads to $p$ occuring before a cycle is closed.

Although the set of accepted traces may still be expressible in MTL, the form of the formula depends on the TS under verification and can be quite unrelated to the original formula (e.g., $\diamond p$ can become $\diamond p \wedge \square(p \Longrightarrow \bigcirc \bigcirc \ldots p)$, where the number of next operators depends on $\mathcal{T}$ ). Only if $L$ is injective with respect to each set of subformulas annotating states of the property automaton (resulting from its construction [28]), can the piggyback algorithm have semantics expressible in logic independently of the transition system, using the original alphabet. But label injectivity is impossible if $\left|2^{A P}\right|<|S|$, because at least two states will map to the same label. For $A P=\{p\}$ this means any system with more than 2 states. We call partial injectivity (i.e., wrt a subset of the codomain of $L$ ) the case when $\varphi$ contains only $p$ and the preimage of $\{p\}$ under $L$ is a singleton. However if both $p$ and $\neg p$ appear in the negated normal form, and $|S|>2$ then $L$ cannot be partially injective wrt to both.

\subsection{Degrees of Liveness}

At this point it is useful to draw a distinction between different levels of liveness, using as example $\neg \varphi=\square \diamond p$. Completely bounded "liveness" as $\square_{k_{1}} \diamond_{k_{2}} p$ is a safety property that constrains a bounded prefix. Period-bounded liveness $\square \diamond_{k} p$ is also a safety property, but over infinite time. If $L$ is partially injective, then piggyback liveness is both a liveness and a safety property, because it can be expressed as $\square \diamond p \wedge \square\left(p \Longrightarrow \diamond_{k-1} p\right)$. So accepting traces include a cycle. The bound constrains from the first occurrence of $p$ and onwards, i.e., the prefix length is not constrained. If $L$ is not partially injective, then piggyback liveness is not expressible in logic. Unbounded liveness does not restrict the cycle length, e.g., $\square \diamond p$ is a qualitative property.

\section{PIGGYBACK AND NEGATION}

Define as $\mathcal{L}_{p}(\varphi)$ the traces of $\mathcal{T}$ accepted by $\varphi$ using the piggyback algorithm, and similarly $\mathcal{L}_{p}(\neg \varphi)$. The verification uses $\neg \varphi$, so $\mathcal{L}_{p}(\neg \varphi)$ is the set of violating traces found

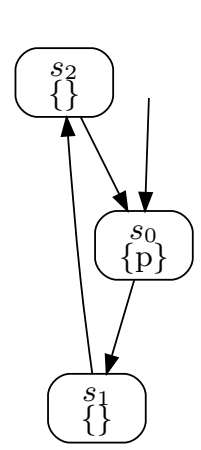

(a) $\mathcal{T}$ (b) $\mathcal{T} \otimes \mathcal{A}_{\varphi}$

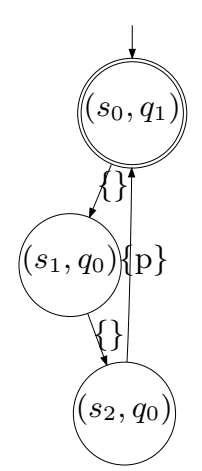

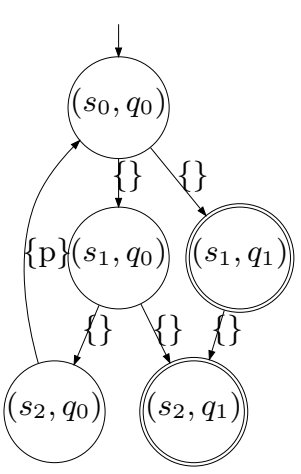

(c) $\mathcal{T} \otimes \mathcal{A}_{\neg \varphi}$
Figure 6: For bound $k=2$ the piggyback algorithm does not find a cycle for neither the property nor its negation.

with the piggyback algorithm. Given $\varphi$ and $\neg \varphi$, a question is whether $\mathcal{L}_{p}(\varphi)$ and $\mathcal{L}_{p}(\neg \varphi)$ are complementary sets, $\mathcal{L}_{p}(\neg \varphi)=\Sigma^{\omega} \backslash \mathcal{L}_{p}(\varphi)$ ? In the rest of this section we prove that this is not the case, so piggyback semantics are not closed under negation. As a counterexample consider the property $\varphi \triangleq \square \diamond p(\neg \varphi=\diamond \square \neg p)$, with BA shown in Fig. 5 . The piggyback algorithm behavior depends on both the formula and the transition system model checked. Consider the transition system $\mathcal{T}$ of Fig. 6a. It is a simple cycle with 3 states, labeled with $\{p\},\{\},\{\}$, and as initial state the one labeled with $\{p\}$. The synchronous products of $\mathcal{T}$ with each of the two Büchi automata are shown in Fig. $6 \mathrm{c}$ and Fig. $6 \mathrm{~b}$. There is no cycle in Fig. 6c, so the piggyback algorithm returns that $\mathcal{T} \forall_{p} \neg \varphi$. Using the piggyback algorithm on Fig. $6 \mathrm{~b}$ with a sufficiently small bound, e.g., $k=2$, fails to detect the accepting cycle. Therefore with $k=2$ the piggyback algorithm would return that $\mathcal{T} \forall_{p} \varphi$. This is inconsistent with negation, it is impossible that both $\varphi$ and $\neg \varphi$ be false for $\mathcal{T}$. It follows that negation and piggyback semantics are not compatible. Selecting $k \geq 3$ would solve this problem, but such a $k_{\text {min }}$ always exists if $\mathcal{T} \otimes \mathcal{A}_{\neg \varphi}$ has finitely many states. An upper bound on $k_{\min }$ is $|\mathcal{T}|\left|\mathcal{A}_{\neg \varphi}\right|$, because no cycle can be longer than this. So $k_{\text {min }}$ depends on both $|\mathcal{T}|$ and $\left|\mathcal{A}_{\neg \varphi}\right|$, thus we can't fix it over the set of all possible $\mathcal{T}$. An arbitrarily large $\mathcal{T}$ can always be constructed by concatenating copies of the accepting cycle from a smaller $\mathcal{T}$. For any formula $\varphi$ and given bound $k$ a transition system can be constructed such that the piggyback algorithm cannot find accepting cycles for neither of $\varphi$ nor $\neg \varphi$. Assuming a non-empty language $\mathcal{L}(\varphi) \neq \emptyset$ there exists some $\mathcal{T}$ that satisfies $\varphi$. So an accepting cycle exists in $\mathcal{T} \otimes \mathcal{A}_{\varphi}$, let $m$ be its length. Concatenating $k$ copies of this cycle we can construct another $\mathcal{T}^{\prime}$, whose accepting cycle cannot be detected by the piggyback algorithm when using bound $k$. Since $\mathcal{T}$ satisfies $\varphi$, an accepting cycle does not exist in $\mathcal{T} \otimes \mathcal{A}_{\neg \varphi}$, neither in $\mathcal{T}^{\prime} \otimes \mathcal{A}_{\neg \varphi}$, because $\mathcal{T}^{\prime}$ still satisfies $\varphi$. As a result, there exists a transition system $\mathcal{T}^{\prime}$, for which the piggyback algorithm cannot find accepting cycles for neither of $\varphi, \neg \varphi$. This proves that in general negation of formulas in the usual way does not correspond to the negation of the property checked by the piggyback algorithm.

\section{ORIGINAL ALGORITHM ISSUES}

In section 3.1 we discussed the issue of injectivity that can prevent the original algorithm from finding counterexam- 


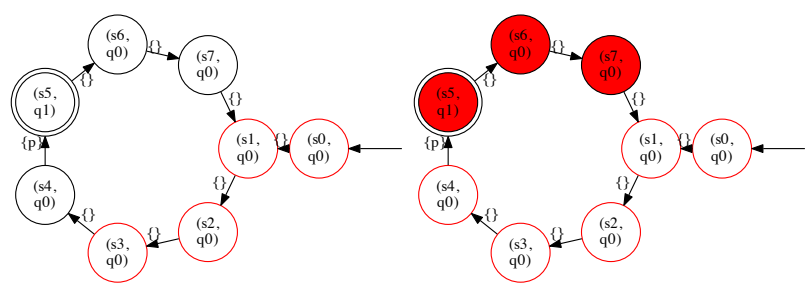

(a)

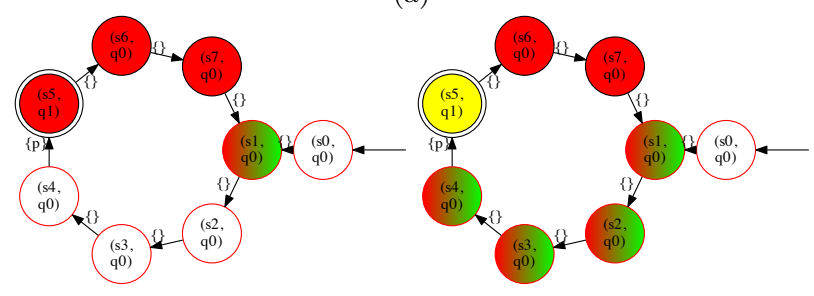

(b)

Figure 7: Without annotating states as visited in free or accepting mode, the original piggyback algorithm would fail to find trivial accepting cycles by blocking at the first visited state.

ples. Our main contribution is addressing it by introducing counter resets in section 6.1. This section considers two other limitations of the original algorithm to motivate further modifications discussed in sections 6.2 and 6.3. By "tip" we will refer to a state in the current BFS front, together with piggybacked information associated with the search when it reaches that state. A tip with no piggybacked accepting states will be referred to as free, otherwise as accepting, also called free and accepting modes.

The original algorithm annotates states in the state space with a bit indicating whether they have been visited in free or accepting mode. This allows revisiting some states at most once, if they are revisited in a mode different than the mode when they were discovered. The reason is demonstrated in Figs. 7a and 7b. Without distinguishing modes, the searching tip piggybacks the accepting state $\left(s_{5}, q_{1}\right)$, but then stops at $\left(s_{7}, q_{0}\right)$ because $\left(s_{1}, q_{0}\right)$ has already been visited. States visited in free mode are marked with colored rims, those visited in accepting mode are filled with a single color and those revisited (so visited in both modes) are filled with two colors. In contrast, in Fig. $7 \mathrm{~b}$ the algorithm continues, because $\left(s_{1}, q_{0}\right)$ to $\left(s_{4}, q_{0}\right)$ have been initially visited in free mode. This enables detecting the accepting cycle, closed at the yellow state. If states are not annotated with an additional bit, then local depth-bounded searching can avoid the issue of Fig. 7a. Local searching is considered in section 6.3 as a means of addressing the issue described in section 5.2, which is of similar nature.

\subsection{Cycle shadowing}

While in accepting mode, the original algorithm ignores any new accepting states discovered. This allows a free tip to potentially visit them later and thus piggyback them. In Fig. 8a the first accepting state piggybacked, $\left(s_{1}, q_{1}\right)$, is also the first visited state re-encountered after traversing the cycle. Therefore the accepting cycle is detected. However, if two accepting states are one after the other, then the first can shadow the second from being discovered. Such cycle shadowing occurs if $k \geq 2$ in Fig. 8b. State $\left(s_{3}, q_{1}\right)$ is ig-

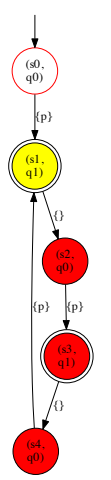

(a)

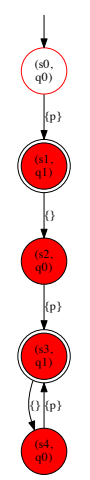

(b)

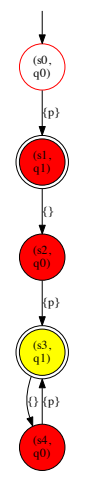

(c)

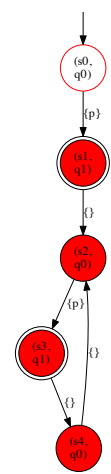

(d)

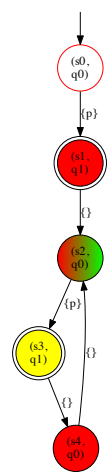

(e)
Figure 8: Shadowing in (b), shadowing and blocking in (d).

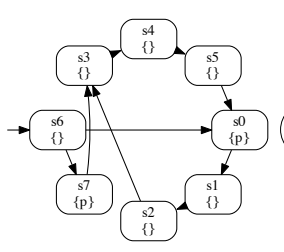

(a) $\mathcal{T}$

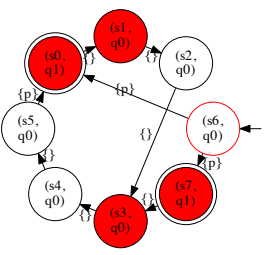

(b)

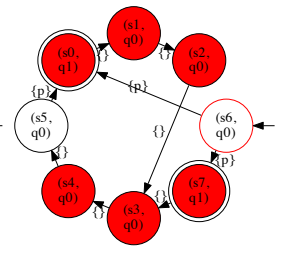

(c)
Figure 9: Searching in $\mathcal{T} \otimes \mathcal{A}_{\neg \varphi}$ for $\neg \varphi \triangleq \square \diamond p$, demonstrating how one tip can block another that has piggybacked a persistent accepting state.

nored because $\left(s_{1}, q_{1}\right)$ is already piggybacked. By storing more piggybacked states as proposed in section 6.2 this issue can be avoided, as shown in Fig. 8c. Fig. 8d shows the combination of shadowing with blocking, analyzed in section 5.2. In this case multi-piggybacking is insufficient and local searching is necessary.

\subsection{Blocking}

Merge points in $\mathcal{T}$ can lead to blocking between different tips, as in Fig. 9, for the following reason. Tip $t_{1}$ piggybacks the accepting state $\left(s_{7}, q_{1}\right)$ and tip $t_{2}$ state $\left(s_{0}, q_{1}\right)$. Then $t_{1}$ leaps to $\left(s_{3}, q_{0}\right)$, in front of $t_{2}$, and marks $\left(s_{3}, q_{0}\right)$ as visited in accepting mode. For bound $k=6$ state $\left(s_{0}, q_{1}\right)$ is on an accepting cycle (so persistent), but $t_{2}$ fails to detect that, because it blocks upon reaching $\left(s_{3}, q_{0}\right)$. On the contrary, tip $t_{1}$ that blocked $t_{2}$ carries $\left(s_{7}, q_{1}\right)$, which is not persistent. This issue can be avoided by triggering local depth-bounded searches whenever a tip reaches a visited state, which is discussed in section 6.3 .

\section{EXTENDED PIGGYBACK ALGORITHM}

The original piggyback algorithm was designed as a minimal extension of reachability analysis to add a limited cycle detection capability. As a result there are cases for which it cannot detect existing accepting cycles shorter than $k$. Lack of labeling injectivity is identified in section 3.1 as one cause. It also renders the set of traces detected by the original algorithm inexpressible in logic, except for special cases. To avoid this we introduce counter resets in section 6.1. In section 5.1 we showed how ignoring accepting states can also lead to missed cycles. It can be avoided by using multi-piggybacking in section 6.2. Blocking between tips is another cause (section 5.2) and can be avoided by 
multi-pjggybacking and local depth-bounded searches (section 6.3).

\subsection{Counter Resets}

\subsubsection{Timed-Automata}

The simplest though substantial change with no added overhead is resetting the counter whenever new accepting states are encountered. Firstly timed-automata are briefly mentioned, to explain the rationale leading to counter resets. Then the piggybacking scheme is augmented with counter resets. Timed-automata are conventional automata equipped with counters [2]. Transition guards can depend on counter values and reset selected counters to zero. We use a discretetime interpretation, for which timed-automata are fully, elementarily decidable [9]. In particular, each automaton derived from some MTL formula is always equivalent to some timed automaton with discrete-time interpretation [2]. At first, timed-automata may appear as a suitable formalism, capable of capturing the counting by the original piggyback algorithm in $\mathcal{T} \otimes \mathcal{A}_{\neg \varphi}$. However this is not the case, because the counter resets are still determined by $\mathcal{A}_{\neg \varphi}$. Each time the product $\mathcal{T} \otimes \mathcal{A}_{\neg \varphi}$ goes through a different accepting state of the same acceptance group $\operatorname{acc}(q)$, the corresponding counter is reset, renewing the horizon over which the search can continue. Therefore, timed Büchi automata cannot express the piggyback algorithm, for exactly the same injectivity-related reasons that untimed Büchi Automata cannot.

\subsubsection{Resets}

The "renewal" behavior of timed automata suggests a modification of the piggyback algorithm to recognize an accepting cycle in $\mathcal{T} \otimes \mathcal{A}_{\neg \varphi}$ whose accepting states belong to the same acceptance group $\operatorname{acc}(q)$. As discussed in section 3.1 $\mathcal{A}_{\neg \varphi}$ observes $\mathcal{T}$ via its AP labels. Consider the simple example of $\neg \varphi \triangleq \square \diamond_{k} p$. This formula is satisfied if $\{p\}$ occurs in a cycle of length less than or equal to $k$. The corresponding timed-automaton $\mathcal{A}_{\neg \varphi}$ has a single accepting state $q_{a}$, a counter $c$ that gets reset upon leaving $q_{a}$, and a guard $c \leq k$ on edges incoming to $q_{a}$. After observing $\{p\}$ the state $q_{a}$ is re-visited if $\mathcal{A}_{\neg \varphi}$ observes $\{p\}$ within $k$ time steps in the future. So visiting $q_{a}$ renews the "waiting time" until observing the next $\{p\}$. This demonstrates how the bound on the search within $\mathcal{T} \otimes \mathcal{A}_{\neg \varphi}$ is updated each time $\mathcal{A}_{\neg \varphi}$ closes a cycle, becoming a receding horizon. So a timed $\mathcal{A}_{\neg \varphi}$ can be utilized and the counter resets be defined from it. In the following we consider primarily properties which are expressible using a single accepting state $q_{j}$ and a single counter $c_{j}$ associated with it, guarding its incoming edges and being reset by its outgoing edges. Some comments on the general case are discussed in section 6.4.1. Note that in any case, if the BA is the untimed version annotated with counters, then any counterexamples found by the extended piggyback algorithm are violations of the untimed property.

Assuming that we have a timed $\mathcal{A}_{\neg \varphi}$ with a single counter $c_{j}$ associated as above to a single accepting state $q_{j}$, then the piggyback algorithm can be modified as outlined in algorithm 1. The counter is initialized to zero and if positive is decremented for each transition traversed. If an accepting state $z_{i j} \triangleq\left(s_{i}, q_{j}\right) \in \mathcal{T} \otimes \mathcal{A}_{\neg \varphi}$ is discovered then $c_{j}=k$ (reset) and if $\left|P_{j}\right|<M$ then $P_{j}:=P_{j} \cup\left\{z_{i j}\right\}$. When $c_{j}$ becomes

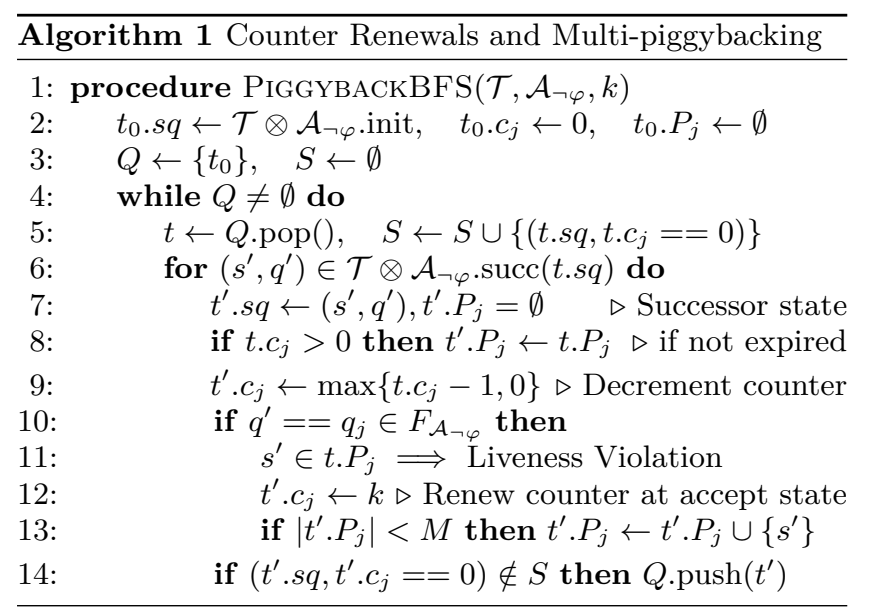

zero, then all piggybacked states are erased, $P_{j}:=\emptyset$. So the search tip "forgets" $P_{j}$ whenever the counter becomes zero. In this section we consider only renewing the counter, so $M=1$ as in [12]. Therefore new accepting states might still not be piggybacked, but unlike the original algorithm, they cause counter resets, so they are not completely ignored. This enables detecting counterexamples as that in Fig. 4 .

\subsection{Multi-Piggybacking}

The modification of section 6.1 addresses the injectivity incompatibility between $\mathcal{A}_{\neg}$ and $\mathcal{T} \otimes \mathcal{A}_{\neg \varphi}$. Next we consider cycle shadowing (section 5.1) that motivates storing all accepting states during exploration. We describe two variants of piggybacking multiple states: exact and lossy. For exact piggybacking $P_{j}$ from section 6.1.2 is implemented with a set of size at most $M$. Increasing $M$ improves the likelihood of detecting an accepting cycle, with unbounded size $M=\infty$ as the extreme. For properties where most states in $\mathcal{T} \otimes \mathcal{A}_{\neg \varphi}$ are accepting, unbounded $M$ is impractical, but for properties where few accepting states exist (e.g., labeled states in a program graph), allowing a larger $M$ is possible. Moreover the accepting states can be piggybacked grouped by acceptance group. For example if $\left(s_{1}, q_{0}\right),\left(s_{3}, q_{0}\right)$ and $\left(s_{15}, q_{1}\right)$ are piggybacked, the it suffices to store $q_{0}$ associated with the set $\left\{s_{1}, s_{3}\right\}$ and $q_{1}$ associated with $\left\{s_{15}\right\}$. Instead of a set, a Bloom filter can be used for $P_{j}$, leading to lossy piggybacking. Scalable Bloom filters [1] can be used to maintain low the error rate. For each acceptance group a different Bloom filter can used, so it suffices to store in the Bloom filter $P_{j}$ only the projection $s_{i}$ of $\left(s_{i}, q_{j}\right)$ on $\mathcal{T}$. Fig. $8 \mathrm{e}$ shows how multi-piggybacking solves the cycle shadowing problem arising in Fig. 8d. If Bloom filters are used, then $\left(s_{i}, q_{j}\right)$ will be matched with some probability of a false positive. Unlike lossy state compression techniques, this is on the safe side: with small probability an accepting cycle may be detected when none exists, but an existing (bounded) accepting cycle cannot be missed. Furthermore, this modification enables detection of every (untimed) counterexample, by never resetting the Bloom filters. In addition, the length of the candidate accepting cycle is at most $k\left|P_{j}\right|$, because each state in $P_{j}$ has been added at most $k$ steps after its preceding addition (otherwise $c_{j}$ would have become zero and $\left.P_{j}:=\emptyset\right)$. Hence performing a $\left(k\left|P_{j}\right|\right)$-bounded reachability search from the matched state can detect hash conflicts. 


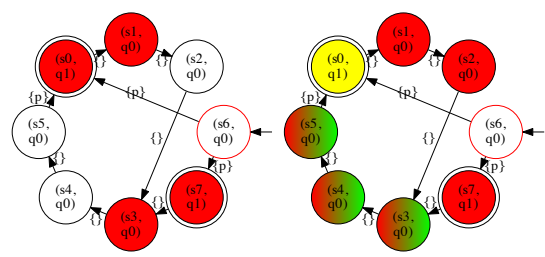

Figure 10: Local depth-bounded DFS can overcome blocking as described in section 6.3.

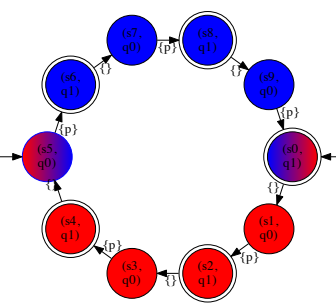

(a)

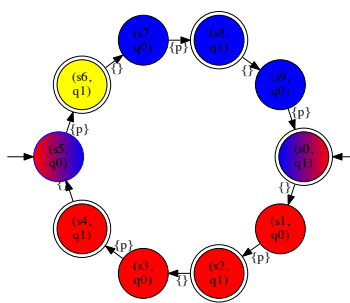

(b)
Figure 11: Instead of blocking as in (a) the search can continues to close the cycle at the yellow state as in (b). This requires local depth bounded DFS and communicating information between searching tips (section 6.3).

\subsection{Gluing}

The multi-piggybacking introduced in section 6.2 can be used also for connecting accepting cycles fragmented among multiple searching tips. The two main differences are performing local, depth $k$-bounded DFS whenever an accepting tip is blocked and storing states as visited in the global state space (as in conventional model checking), instead of annotating them with their visit mode (accepting or free). The local searches incur a cost, whereas maintaining a state space without annotation reduces the re-exploration that can occur with the original algorithm. We describe the local DFS, for a single accepting state $q_{j} \in \mathcal{A}_{\neg \varphi}$. If a search tip $t_{r}$ has nothing piggybacked and reaches an explored state $z \in \mathcal{T} \otimes \mathcal{A}_{\neg \varphi}$, then it stops because that transition does not belong to an accepting cycle (for the bounded-suffix property). If $t_{r}$ does have piggybacked states, $P_{j}^{r} \neq \emptyset$, then local DFS is performed up to depth $k$ relative to $z$. The search truncates whenever an accepting state is found or the current BFS front is reached (analyzed later). Thus each local DFS detects the front $A_{k}(z)$ of accepting states reachable in at most $k$ steps from the state $z$ that blocked $t_{r}$. If $A_{k}(z) \cap P_{j}^{r} \neq \emptyset$ then an accepting cycle has been closed. If $A_{k}(z)=\emptyset$, then $t_{r}$ is discarded, because no (bounded-suffix) cycle can include the blocking state $z$. Otherwise each accepting state $z^{\prime} \in A_{k}(z)$ can be used to forward the piggybacked information to those tips of the BFS front that are still active. To achieve this, between BFS iterations, each active tip $t_{w}$ needs to check if $z^{\prime} \in P_{j}^{w}$, for each $z^{\prime}$ resulting from blocking in the last BFS iteration. Note that this is possible also with lossy piggybacking, i.e., when each $P_{j}^{w}$ is implemented using a Bloom filter. In addition, to account for cases when tip $z^{\prime}$ has been piggybacked by another tip $t_{u}$ that is blocked in the same BFS iteration, firstly $P_{j}^{r}$ need to be forwarded from each blocked tip $t_{r}$ to any other tip $t_{u}$ that blocked in the same iteration and is reachable through forwarding from $t_{r}$. After this is complete, forwarding to active tips can be computed. When the local DFS truncates at states that belong to the current BFS front, then it can annotate that state by storing a pointer to its piggybacked information. In the next iteration, the single tip that is currently at that state can first check any pointers annotating the state, retrieve the linked piggybacked information (which can now be deleted) and then continue the search. Note that for problems with a large ratio of accepting states in $\mathcal{T} \otimes \mathcal{A}_{\neg \varphi}$ this approach can trigger a prohibitively large number of local searches and forwarding can result in high overhead between BFS iterations. Compared to previous approaches, gluing to some extent resembles back-level edges [6], but with the second stage replaced by bounded searches which are interleaved with the state space exploration. An alternative is to store the blocked tuples $\left(P_{j}^{w}, A_{k}(z)\right)$ of piggybacked accepting states and $k$-reachable accepting states, then at the end construct a smaller directed graph, comprised only of accepting states, and check for cycles in that acceptance graph.

\subsection{Bounded-Suffix LTL Properties}

For certain properties there exists some lower bound $k_{\min }$, such that selecting $k \geq k_{\min }$ leads to the piggyback algorithm verifying the original formula. More interestingly, the formulas with this property include several of the most commonly used ones in verification [19]. The universal versions of some examples are: $\square p, \diamond p, \square \diamond p, p \mathcal{U} q, \neg(p \mathcal{U} q)$ and $\diamond \square_{k} p$. A simple example can demonstrate this: consider the property $\forall \square \diamond p$, whose negation is $\exists \diamond \square \neg p$. The equivalent $\mathcal{A}_{\neg \varphi}$ has a single accepting state with a self-loop. Intuitively, this means that as soon as it detects $\{p\}$ in $\mathcal{T}$, the accepting cycle in $\mathcal{T} \otimes \mathcal{A}_{\neg \varphi}$ is interrupted. There does not exist an accepting cycle that includes any state labeled with $\{p\}$. This provides us with a tight upper bound on the guard, in this case $k=1$. Choosing $k \geq 1$ implies that we perform full LTL model checking for $\square \diamond p$. More generally, if $q$ is an accepting state of $\mathcal{A}_{\neg \varphi}$ and the largest cycle through $q$ is of finite length $L$, then the extended piggyback algorithm with bound $k \geq L$ checks the validity of the given formula. The key property above is the bound on accepting cycle length. Note that we refer to the largest cycle, not the largest simple cycle, which is an NP-hard problem and not relevant to our case. If a non-simple cycle exists, then an infinite cycle exists and the above property does not hold. Deciding whether an infinite accepting cycle exists, or if not, finding the accepting circumference of $\mathcal{A}_{\neg \varphi}$ can be solved with a BFS in time linear in $\left|\mathcal{A}_{\neg \varphi}\right|$. In practice the automaton $\mathcal{A}_{\neg \varphi}$ is small, so finding the accepting circumference and if finite using it as bound for the piggyback algorithm is a computationally cheap preprocessing stage. So properties that satisfy the above boundedness condition can be fully checked provided counter resets, multi-piggybacking and gluing are all used. Note that the "good" properties in [15] have bounded suffix. In contrast properties like $\forall \diamond \square p$ are not included, because the negation $\exists \square \diamond \neg p$ may have an arbitrarily large suffix (cycle length). For a fixed $\mathcal{T}$ the counterexample suffix is always finite, but depends on $\mathcal{T}$ (which can be arbitrary). The piggyback algorithm can also be adapted for the class of persistence never claims, which are expressible by weak Büchi Automata [26]. If $\mathcal{A}_{\neg \varphi}$ is a weak automaton, then any accepting cycle includes only accepting states from a subset of $\mathcal{A}_{\neg \varphi}$ states, so the counter resets can be adapted to monitor the subsets of $Q_{\mathcal{A}_{\neg \varphi}}$, with bound $k=1$. Moreover, if forwarding is used, then each local DFS has depth bound 1. 


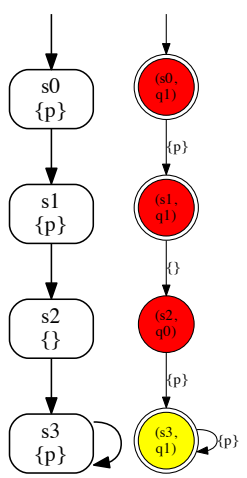

(a)

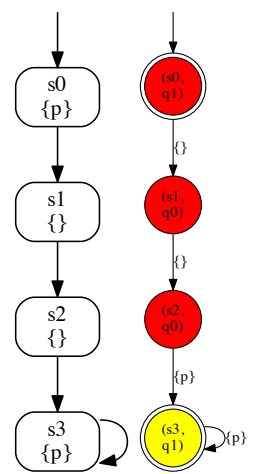

(b)

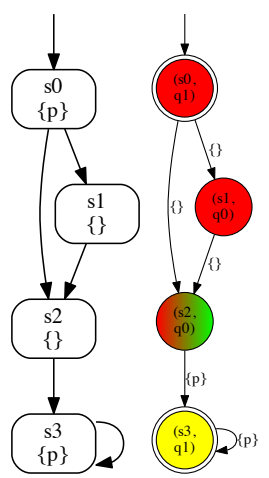

(c)
Figure 12: The piggyback algorithm can find the accepting cycle in all cases, though the prefix does not satisfy $\square \diamond_{1} p$ for (b). Nonetheless it does satisfy $\square \diamond p$ (unbounded liveness).

\subsubsection{Multiple Counters}

Here we have considered adding counters to automata obtained from translating LTL, primarily as an aid motivating the counter resets introduced to the algorithm. So the timed automata have the same states and edge annotations as their corresponding untimed versions. Therefore any accepting cycle in the timed automaton is an accepting cycle for the untimed automaton, even if it violates some guards. Also, we have restricted our attention to simple cases with a single accepting state and one clock associated to it, guarding its entries and being reset upon leaving it. The general case of translating LTL to timed automata is beyond the scope of the present paper. It concerns relating the bounds on eventualities of $\neg \varphi$ to the clocks of accepting states in $\mathcal{A}_{\neg \varphi}$. If each clock is not associated only with a single accepting state, then the situation becomes complicated and the piggyback algorithm as extended here may not verify the original property.

\subsubsection{Accepting Cycle Reachability}

For simple cases with a single accepting state and timed $\mathcal{A}_{\neg \varphi}$ resulting by addition of clocks to the untimed version, the piggyback algorithm with resets, multi-piggybacking and gluing does not miss any trace accepted by the timed $\mathcal{A}_{\neg \varphi}$ and in addition can detect some traces rejected by the timed $\mathcal{A}_{\neg \varphi}$, but accepted by its untimed version (equiv. the timed version with longer bound). A simple example is given in Fig. 12, where the accepting cycle is found in all three cases by the piggyback algorithm, but only (a) and (c) are satisfy $\neg \varphi=\square \diamond_{1} p$. The reason is that although the cycle in (b) is accepting, it is not reachable from the initial state. The piggyback algorithm can be restricted to check only the timed version, but there is no loss in allowing it to (opportunistically) detect more counterexamples. Bounding the liveness in $\neg \varphi$ yields an underapproximation of the set of counterexamples that the piggyback algorithm can detect.

\section{EXPERIMENTS}

This section includes results using the piggyback algorithm with counter resets (section 6.1.2) but not multi-piggybacking or gluing. We use examples from the the BEEM set of benchmarks [21]: anderson 5 [4] with partial order reduction (POR), and bakery. 5 [17] and lamport. 8 [18] without POR. Note that partial order reduction is conservative with respect to bounded liveness, because it increases the length of cycles that can be detected. The distribution of states with respect to depth is shown in Fig. 18. The hardware used has Intel(R) Xeon ${ }^{\circledR}$ X5550 processors with a total of 16 processing cores, 12 GB RAM and runs Ubuntu 10.04.4. The properties verified are $\varphi_{1}=\square \diamond\left(P_{2} @ C S\right)$ for anderson.5, $\varphi_{2}=\square\left(\neg P_{0} @ C S \Longrightarrow \diamond P_{0} @ C S\right)$ for lamport.8 and bakery.5. The negation of $\varphi_{1}$ is a bounded-suffix property, with bound $k=1$ (depending on the exact details of decrementing in the implementation before or after leaving the state where a state was piggybacked, the bound in code can differ by 1). The negation $\neg P_{2} @ C S$ includes all states of $P_{2}$ other than the critical section, so most states of $\mathcal{T} \otimes \mathcal{A}_{\neg \varphi}$ are accepting. Therefore renewals occur frequently and maintain an accepting state which was piggybacked early in the search. Although without multi-piggybacking this can lead to shadowing (section 5.1) the algorithm can still find counterexamples. Using a sufficient bound, for bakery. 5 and lamport. 8 counterexamples are found by every run, whereas for anderson.5 they are found with a frequency of $70 \%$, because interleaving between cores can lead to cases that would need the extensions discussed in section 6.2 and section 6.3. For anderson.5 only for $k \geq 40$ does the original algorithm find counterexamples, because a counterexample involves a single process cycling through all the 5 Slots used for implementing the mutual exclusion. Note that all counterexamples are unfair. With weak fairness enabled, SPIN depth-first search cannot find counterexamples for anderson.5, verifying the claims in [4]. Using different bounds can affect the number of states stored in different modes (accepting or free), but for the particular property $\varphi_{1}$ a large number of states are accepting, therefore any free tip quickly encounters and piggybacks some accepting state, thus the selected bound $k$ does not have an observable effect on running time. For anderson. 5 Figs. 13 and 14 show comparisons of running time wrt number of cores between the original piggyback algorithm and its extension with counter renewals. For bound $k=1$ the original algorithm does not find any counterexamples. For bound $k=100$ each run in these graphs did find one or more counterexamples. Performance remains unaffected after introducing counter renewals, as demonstrated by the proximity between the different curves. The difference is that for bound $k=1$ the new version with renewals does find counterexamples, whereas the original piggyback algorithm requires a bound of $k=40$ and more to find counterexamples. This is caused by the fact that the original algorithm searches for cycles of fixed length $k=40$ in the product automaton $\mathcal{T} \otimes \mathcal{A}_{\neg \varphi}$. Therefore for any bound, the original algorithm requires that the user either have a priori knowledge about the model and anticipate what size of cycle to look for, or that they iteratively adjust the bound, while searching for a cycle. On the contrary, the proposed improvement decouples the bound from the product, and binds it only to the property $\mathcal{A}_{\neg \varphi}$. Thus firstly it renders the language accepted by the algorithm expressible in logic, secondly what bound to use relates primarily to the automaton (e.g., whether its language includes only bounded-suffix words) and not to the specific transition system that is being verified. Fig. 15 is the distribution of liveness checks using counter renewals and bound $k=1$ that terminate upon detecting a counterexample, as represented by their running time and depth reached. Note that this version of the algorithm, which includes renewals but not 


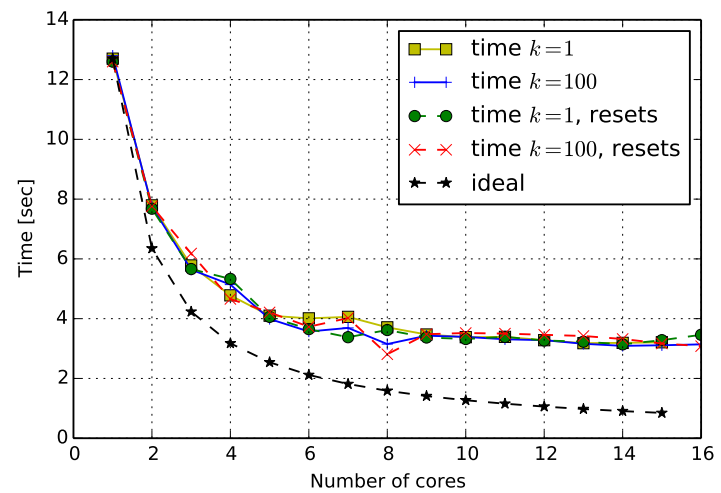

Figure 13: Comparison of counter renewals to original piggyback algorithm for the anderson. 5 example. These runs continue until covering the state space.

transferring information between different tips, only counterexamples of (prefix plus suffix) length less than the BFS tree depth can be found. So the depth reached is also the length of the counterexample found.

By negating the desired property to $\varphi_{3}=\neg \square \diamond\left(P_{2} @ C S\right)$ we obtain a $\mathcal{A}_{\neg \varphi}$ whose accepting cycles do not admit an upper bound, i.e., a property whose counterexamples do not have bounded suffixes. Therefore it is expected that the bound now depends on the transition system, because the next accepting product state can delay to appear in $\mathcal{T} \otimes \mathcal{A}_{\neg \varphi}$ arbitrarily long. Running the original algorithm requires bound at least $k=27$ to find counterexamples. The counterexample previously consisted of a single process other than $P_{2}$ cycling through all the Slots. In this case the counterexamples consist of $P_{2}$, possibly together with some other process, cycling through all the Slots, thus infinitely often visiting its critical section. Running the revised algorithm finds counterexamples as soon as we select the bound $k=5$. The reason is that there does exist a counterexample, namely the one where $P_{2}$ alone cycles through all the Slots, in which an accepting state is encountered every 5 transitions, because this is the number of states included in a single do iteration of process $P_{2}$. This demonstrates how despite its dependence in this case on $\mathcal{T}$, still a lower bound is made possible by using counter renewals. Finally it is worth noting that the set of accepting states $F_{\mathcal{T} \otimes \mathcal{A}_{\neg \varphi}}$ for $\varphi_{3}=\neg \square \diamond\left(P_{2} @ C S\right)$ is the complement of the accepting states of $\varphi_{1}$ in this section. This leads to less frequent piggybacking, hence reduced dependence on the interference between different search tips, and as a result we observe frequency of finding counterexamples for anderson. 5 (given sufficient bound) close to $100 \%$ for this case.

Fig. 16 compares results for lamport. 8 with the original piggyback algorithm that needs $k \geq 8$ to find counterexamples, to its extension with counter renewals that can find counterexamples with $k=1$. The results from bakery. 5 are shown in Fig. 17, where the running times of runs that terminate upon fining an accepting cycle have been grouped by the total depth of the prefix and suffix (depth at which the cycle is closed). Note that the depths reported by SPIN v6.2.7 or earlier account also for never claim moves, thus they differ from the corresponding BFS tree depths by a factor of 2 .

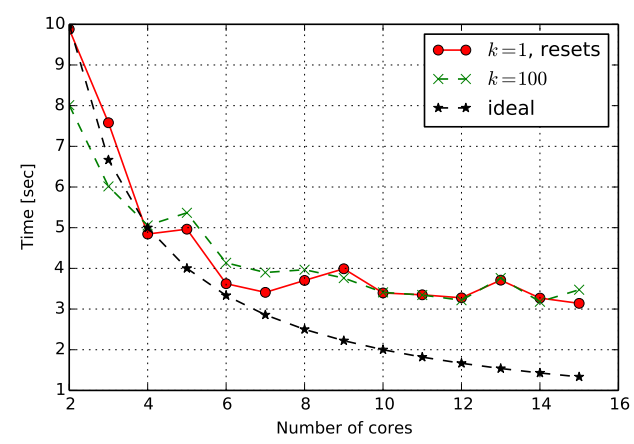

Figure 14: Comparison of counter renewals to original algorithm for anderson. 5 stopping at the first cycle found and averaging times over multiple runs.

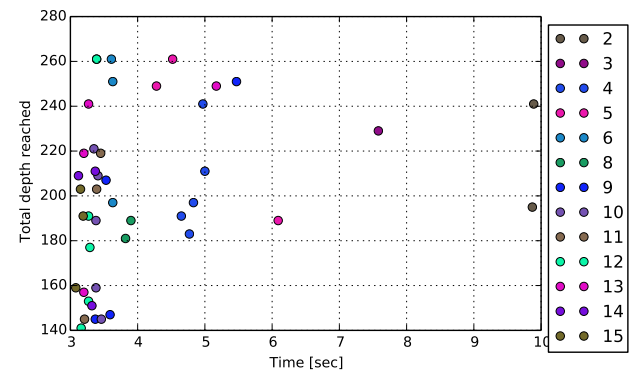

Figure 15: Counterexample depth wrt running time for liveness checks of anderson. 5 with bound $k=1$ and counter renewals.

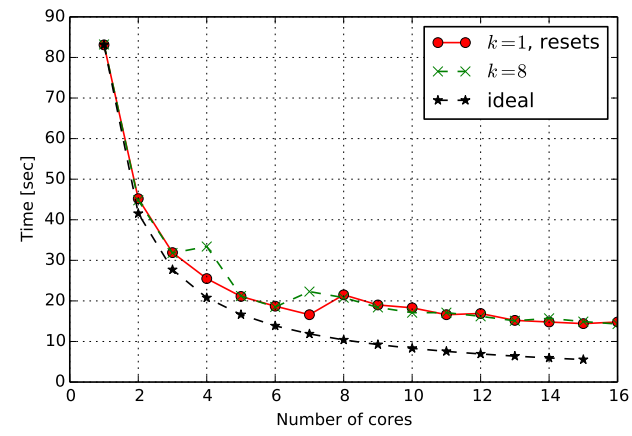

Figure 16: Comparison of counter renewals to original algorithm for lamport.8, the runs continue until covering the state space.

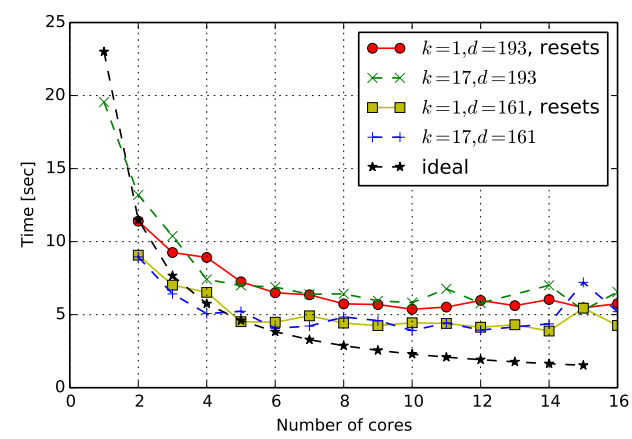

Figure 17: Comparison of counter renewals to original algorithm for bakery.5, stopping at the first cycle found. 


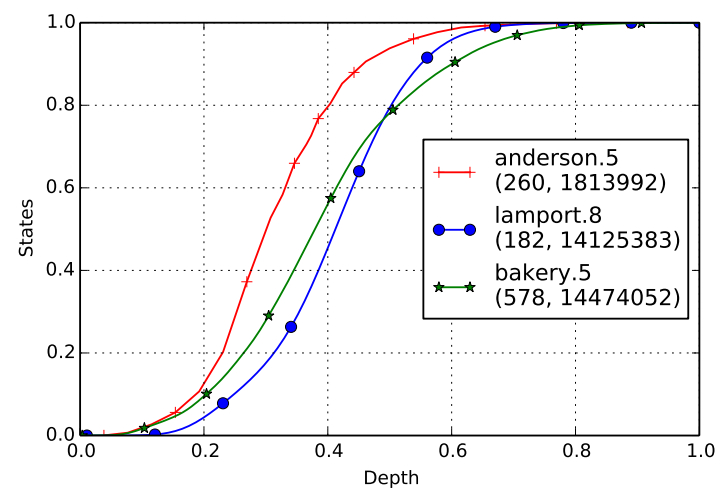

Figure 18: Normalized number of states with respect to depth. BFS depth and number of states in parentheses.

\section{PARALLEL MODEL CHECKING}

This section reviews some results from P-completeness theory to motivate the effort of parallelizing model checking for a given automaton. Sublinear parallel algorithms differ drastically from sequential ones, in that they are top-down. They process the whole state space in a brute force manner, including unreachable states. Even if a parallel solution processing only the reachable states exists, it would still lead to a very large problem size. Nonetheless the value is in showing that finding accepting cycles in $\mathcal{T} \otimes \mathcal{A}_{\neg \varphi}$ is highly parallelizable and not inherently sequential. Another limitation is the cubic number of processors required (best known bound above $2[27])$. The usual assumption that input is already present in shared memory upon initialization of the algorithm can be circumvented by first distributing a compact symbolic problem definition, then generate the transition relation of $\mathcal{T} \otimes \mathcal{A}_{\neg \varphi}$ and store it in shared memory in time $O(1)$ using $N^{2}$ processors.

\subsection{High Parallelizability}

Using multiple processors for computation can potentially reduce the required time. Problems decidable in polynomial time (class P) are considered as highly parallelizable if they can be solved in poly-logarithmic time $\log ^{O(1)} N$ on a number of processors polynomial in $N$, known as NC (Nick's class) and it is widely believed that $\mathrm{NC} \neq \mathrm{P}[25,8]$. For each $k$ the corresponding subclass $O\left(\log ^{k} N\right)$ is known as $\mathrm{NC}^{k}$, so $\mathrm{NC}=\bigcup_{i \in \mathbb{N}} \mathrm{NC}^{i}$, and $\mathrm{NC}^{i} \subseteq \mathrm{NC}^{i+1}$, defining the NC-hierarchy. By the space hierarchy theorem it holds that NC $\neq$ PSPACE (p.70 [8]). The following relations among complexity classes hold $\mathrm{NC}^{1} \subseteq \mathrm{L}=\mathrm{SL} \subseteq \mathrm{NL} \subseteq \mathrm{NC}^{2} \subseteq$ $\mathrm{NC} \subseteq \mathrm{P} \subseteq$ PSPACE, where SL is the class of problems reducible to undirected graph connectivity (USTCON) [23] and NL the problems decidable in non-deterministic logarithmic time. Directed graph connectivity (STCON) is NL-complete (Thm.16.2 [20]).

\subsection{Persistence Checking in NC}

The existential version of model checking concerns deciding whether a counterexample exists or not, whereas the constructive version requires finding a counterexample, if one exists. Both the constructive and the existential versions of LTL model checking are PSPACE-complete, Thm.5.46 [5]. Therefore LTL model checking is not in NC. Nonetheless the cause is the translation of LTL formulas to Büchi Automata. With respect to the transition system size (i.e., for a fixed formula) model checking is in $\mathrm{P}$ and as will be shown next, it is also in NC. The automata theoretic form of the model checking problem consists of detecting an accepting cycle in the synchronous product $\mathcal{T} \otimes \mathcal{A}_{\neg \varphi}$. Lexicographic Depth-First Search (DFS) has been proved to be P-complete [22]. So using nested DFS [13] for persistence checking (Thm 4.65 [5]) cannot be expected to scale satisfactorily with the number of processors. Despite this, it is shown next that persistence checking is in NC.

\subsubsection{Transitive Closure}

As noted above, the directed graph reachability problem is NL-complete, so in $\mathrm{NC}^{2}$ (p.362 and Thm.16.2 [20]). We summarize here the $\mathrm{NC}$ computation of the transitive closure $A^{*}$ of a relation $A \in \mathbb{B}^{N \times N}$ where $\mathbb{B} \triangleq\{0,1\}$ (here $A$ is the adjacency of the product $\left.\mathcal{T} \otimes \mathcal{A}_{\neg \varphi}\right)$. The reflexive transitive closure $\bar{A} \triangleq(I \vee A)^{*}=(I \vee A)^{N}$ can be computed in time $O\left(\log ^{2} N\right)$ by $N^{3}$ processors (p.212 [20]). Starting with $I \vee A$, it is squared in each iteration, thus requiring $\lceil\log N\rceil$ iterations to compute $(I \vee A)^{2^{\lceil\log N\rceil}}$. Each iteration involves a matrix multiplication, which needs $N^{2}$ rowcolumn vector multiplications. Each vector multiplication $a^{\mathrm{T}} b=\bigvee_{i=1}^{N} a_{i} \wedge b_{i}$ is computable in time $O(\log N)$ by iteratively halving the conjuncts using $N$ processors. Overall time $O\left(\log ^{2} N\right)$ and $N^{3}$ processors are used. The transitive closure $A^{*}$ has the same off-diagonal elements as the reflexive transitive closure $(I \vee A)^{*}$, i.e., $\neg I \wedge A^{*}=\neg I \wedge(I \vee A)^{*}$. However $\operatorname{diag}\left(A^{*}\right) \neq \operatorname{diag}\left((I \vee A)^{*}\right)$. To construct the diagonal of $A^{*}$ we observe that $A_{i i}^{*}=1$ if and only if (iff) state $q_{i}$ has a self-loop or is on a cycle of length larger than one. A state $q_{i}$ has a self-loop iff $A_{i i}=1$. It is on a cycle larger than one if it can reach some state $q_{j}$ from which it is reachable, i.e., $A_{i j}^{*} \wedge A_{j i}^{*}=1$ for any $j \neq i$, equivalently $\bigvee_{j \neq i} A_{i j}^{*} \wedge A_{j i}^{*}=1$, collectively the $\operatorname{diag}\left(\left(\neg I \wedge A^{*}\right)^{2}\right)$. But as noted earlier the off-diagonal elements of $A^{*}$ are those of $(I \vee A)^{*}$, so $\left(\neg I \wedge A^{*}\right)^{2}=\left(\neg I \wedge(I \vee A)^{*}\right)^{2}$. Thus the transitive closure $A^{*}=A \vee(\neg I \wedge \bar{A}) \vee(\neg I \wedge \bar{A})^{2}$, where $A$ captures the self-loops (its off-diagonal nonzero elements are included in $\bar{A}),(\neg I \wedge \bar{A})$ answers all reachability queries between different states and $(\neg I \wedge \bar{A})$ captures states that are on cycles longer than one. The conjunctions, disjunctions and negations of square matrices are each computable in time $O(1)$ with at most $N^{2}$ processors. The matrix multiplication to obtain $(\neg I \wedge \bar{A})^{2}$ requires time $O(\log N)$ on $N^{3}$ processors. So overall $\bar{A}$ and $A^{*}$ require time $O\left(\log ^{2} N\right)$ on $N^{3}$ processors. Also $\operatorname{diag}(\bar{A})=I$, so $\bar{A}=I \vee A^{*}$, a result we use later.

\subsubsection{Existence of Reachable Accepting Cycles}

Define $a^{\mathrm{T}} b \triangleq \bigvee_{i=1}^{n} a_{i} \wedge b_{i}$ and $\left(a b^{\mathrm{T}}\right)_{i j} \triangleq a_{i} \wedge b_{j}$. Answering the persistence problem requires finding whether there exists an accepting state that is both reachable from an initial state and on a cycle. These two subproblems can be answered using the transitive closure $A^{*}$ as follows. Let $\beta \in \mathbb{B}^{n}$ have $\beta_{i}=1$ if $q_{i}$ is an accepting state on a cycle, $\beta_{i}=0$ otherwise. Let $\gamma \in \mathbb{B}^{n}$ have $\gamma_{i}=1$ if $q_{i}$ is an initial state, $\gamma_{i}=0$ otherwise. The matrix $B \triangleq \gamma \beta^{\mathrm{T}}$ has $B_{i j}=1$ iff $q_{i}$ is an initial state and $q_{j}$ a persistent accepting state. The conjunction $W \triangleq B \wedge\left(I \vee A^{*}\right)=B \wedge \bar{A}$ has $W_{i i}=1$ iff $q_{i}$ is an initial and a persistent accepting state, and $W_{i j}=1, i \neq j$ iff the initial state $q_{i}$ can reach the persistent accepting state $q_{j}$ (after one or more tran- 
sitions). So $W_{i j}=1$ iff the initial state $q_{i}$ can reach the persistent accepting state $q_{j}$ after 0 or more transitions. It remains to compute which accepting states are persistent, i.e., $\beta$. A state is on a cycle iff it is reachable from itself after one or more transitions (but not zero). The diagonal $\operatorname{diag}\left(A^{*}\right)$ has ones at persistent states. If $\zeta \in \mathbb{B}^{N}$ has ones at accepting states, then $\beta=\zeta \wedge \operatorname{diag}\left(A^{*}\right)$, which solves the second subproblem. By combining the previous results there exists a persistent accepting state reachable from some initial state, iff $W$ contains a nonzero element, equivalently iff $\bigvee_{i=1}^{n} \bigvee_{j=1}^{n} W_{i j}$. The disjunction of $N^{2}$ matrix elements can be computed in time $O\left(\log \left(N^{2}\right)\right)=O(2 \log N)=O(\log N)$ by $N^{2}$ processors that halve the number of conjuncts in each iteration, each processor performing a conjunction between a pair. It is $W=\left(\gamma \beta^{\mathrm{T}}\right) \wedge \bar{A}=\gamma\left(\zeta \wedge \operatorname{diag}\left(A^{*}\right)\right)^{\mathrm{T}} \wedge \bar{A}$. The vector exterior product requires time $O(1)$ on $N^{2}$ processors. As discussed in section 8.2.1 computing $A^{*}$ and $\bar{A}$ requires time $O\left(\log ^{2} N\right)$ on $N^{3}$ processors. Note how checking existence of a counterexample has been shown to be in $\mathrm{NC}$, however explicit construction of the counterexample is unlikely to be so, because it involves ordering the states. Note that translation from LTL to automata is a separate stage. In practice formulas and their associated automata are relatively small, whereas the limiting factor is primarily $|\mathcal{T}|$ (p.293 [5]).

\section{CONCLUSIONS AND FUTURE WORK}

We presented an improvement of the piggyback algorithm for parallel model checking that increases the subset of violating traces it can detect. The main change is resetting the counters whenever visiting a product state that projects on the same accepting state of the automaton as the piggybacked accepting product state. Other modifications we propose concern piggybacking multiple states, a conservative version using Bloom filters and connecting pieces of cycles by means of local depth-bounded searches that are triggered when visited states are revisited.

\section{ACKNOWLEDGMENTS}

This work was supported by a Graduate Research Fellowship from the Jet Propulsion Laboratory, California Institute of Technology.

\section{REFERENCES}

[1] P. S. Almeida, C. Baquero, N. Preguiça, and D. Hutchison. Scalable bloom filters. Inf. Process. Lett., 101(6):255-261, 2007.

[2] R. Alur and D. L. Dill. A theory of timed automata. Theor. Comput. Sci., 126(2):183-235, 1994.

[3] R. Alur, K. Etessami, S. La Torre, and D. Peled. Parametric temporal logic for "model measuring". ACM Trans. Comput. Logic, 2(3):388-407, 2001.

[4] J. H. Anderson, Y.-J. Kim, and T. Herman. Shared-memory mutual exclusion: major research trends since 1986. Distr. Comp., 16(2-3):75-110, 2003.

[5] C. Baier and J.-P. Katoen. Principles of Model Checking. The MIT Press, 2008.

[6] J. Barnat, L. Brim, and I. Černá. Cluster-based ltl model checking of large systems. FMCO'05, pages 259-279, 2005.

[7] E. Clarke, A. Biere, R. Raimi, and Y. Zhu. Bounded model checking using satisfiability solving. Formal
Methods in System Design, 19(1):7-34, 2001.

[8] R. Greenlaw, H. J. Hoover, and W. L. Ruzzo. Limits to Parallel Computation: P-completeness Theory. Oxford Univ. Press, 1995.

[9] T. A. Henzinger, J.-F. Raskin, and P.-Y. Schobbens. Temporal logics, automata, and classical theories for defining real-time languages. Technical Report UCB/CSD-99-1074, Berkeley, 1999.

[10] G. Holzmann. The model checker spin. Software Eng., IEEE Trans. on, 23(5):279-295, 1997.

[11] G. Holzmann and D. Bosnacki. The design of a multicore extension of the spin model checker. Softw. Eng., IEEE Trans. on, 33(10):659-674, 2007.

[12] G. J. Holzmann. Parallelizing the spin model checker. In Proc. 19th Intl. Conf. on Model Checking Softw., SPIN'12, pages 155-171, 2012.

[13] G. J. Holzmann, D. Peled, and M. Yannakakis. On nested depth first search. SPIN'96, 32:81-89, 1996.

[14] R. Koymans. Specifying real-time properties with metric temporal logic. Real-Time Systems, 2(4):255-299, 1990.

[15] O. Kupferman, N. Piterman, and M. Y. Vardi. From liveness to promptness. In Proc. 19th Int. Conf. on Comp. Aided Verif., CAV'07, pages 406-406, 2007.

[16] A. Laarman, R. Langerak, J. Van De Pol, M. Weber, and A. Wijs. Multi-core nested depth-first search. In Proc. 9th Intl Conf. on Autom. Tech. for Verif. and Anal., ATVA'11, pages 321-335, 2011.

[17] L. Lamport. A new solution of dijkstra's concurrent programming problem. Comm. ACM, 17(8):453-455, 1974.

[18] L. Lamport. A fast mutual exclusion algorithm. ACM Trans. Comput. Syst., 5(1):1-11, 1987.

[19] Z. Manna and A. Pnueli. Tools and rules for the practicing verifier. Technical report, Stanford, Stanford, CA, USA, 1990.

[20] C. H. Papadimitriou. Computational complexity. Addison-Wesley, 1994.

[21] R. Pelánek. Beem: Benchmarks for explicit model checkers. In Proc. 14th SPIN Conf. on Model Checking Softw., pages 263-267, 2007.

[22] J. H. Reif. Depth-first search is inherently sequential. Information Processing Letters, 20(5):229 - 234, 1985.

[23] O. Reingold. Undirected connectivity in log-space. J. ACM, 55(4):17:1-17:24, 2008.

[24] V. Schuppan and A. Biere. Efficient reduction of finite state model checking to reachability analysis. Int. J. Softw. Tools Technol. Transf., 5(2):185-204, 2004.

[25] M. Sipser. Introduction to the Theory of Computation. Cengage Learning, 3 edition, 2013.

[26] I. Černá and R. Pelánek. Relating hierarchy of linear temporal properties to model checking. Math. Found. Comp. Sc., 2747:318-327, 2003.

[27] V. V. Williams. Multiplying matrices faster than coppersmith-winograd. In Proc. 44th Ann. ACM Symp. Theory of Comp., STOC '12, pages 887-898, 2012.

[28] P. Wolper. Constructing automata from temporal logic formulas: a tutorial, volume 2090, pages 261-277. LNCS, 2002. 